\title{
Hysteretic control of grid-side current for a single-phase LCL grid-connected voltage source converter
}

\author{
Santiago Cóbreces ${ }^{\mathrm{a}, *}$, Robert Griñó ${ }^{\mathrm{b}}$ \\ ${ }^{a}$ GEISER. Deparment of Electronics. Escuela Politécnica Superior. Universidad de Alcalá. \\ Alcalá de Henares. Spain \\ ${ }^{b}$ Institute of Industrial and Control Engineering. Universitat Politècnica de Catalunya. \\ Barcelona. Spain
}

\begin{abstract}
This paper proposes a new approach to control the grid-side current of LCL-grid connected voltage source converters using hysteretic relay feedback controllers. The closed loop system is stabilized by designing a local feedback around the relay element. The compensator allows the use of relay feedback controllers by making the controlled plant almost strictly positive real. The article proposes the use of the locus of the perturbed relay system as analysis and design tool and studies orbital stability for several plant and controller conditions. The approach is validated by means of simulation testing.
\end{abstract}

Keywords: AC/DC, hysteresis, LCL filter, ASPR, parallel compensation

\section{Introduction}

Hysteretic controlled systems in particular and, in general, Relay Feedback Systems (RFS), are among the oldest and most spread control schemes in the world. The feedback loops controlled in that way present a high degree of 5 simplicity, a superb performance and wide robustness margins. A good proof of that is their wide presence in quotidian systems as home heaters, electrical appliances, etc.

\footnotetext{
* Corresponding author

Email addresses: cobreces@depeca.uah.es (Santiago Cóbreces), roberto.grino@upc.edu (Robert Griñó)
}

Preprint submitted to Mathematics and Computers in Simulation 
The use of RFS is also extended in the control of power electronics systems. Their advantages have been proved to be useful in AC machine current control [17, 15, 5], and grid-connected current control, in both SISO and MIMO plants [21, 7, 14]. A common feature of these proposals is the resistive-inductive dynamical characteristic, from the current control point of view, of the system the power converter is facing, being it a consequence of the RL EMI filter or of the equivalent circuit of, for example, an electric machine. The simplicity of this structure perfectly suits the relay feedback behaviour creating control loops with outstanding robustness to grid, load or filter parameter mismatching, lack of current tracking errors, and very fast dynamics only limited by plant bandwidth. Although the use of RL filters was standard in the early ages of the connection of VSCs to the grid, the use of higher order filters as, for example,

2o LCL filters is increasingly gaining presence in grid applications. In the latter cases the application of RFS presents several problems and there are only proposals to control the converter-side current, such as [20] or [3], or the whole state vector as is the case in the related approach based on sliding control schemes [9, 23, 10].

This fact is probably caused by the interesting dynamical characteristics of the transfer function relating converter-side current and converter output voltage: it is and admittance transfer function, and it is well known that impedance or admittance transfer functions are positive real (PR)[1]. That is to say

$$
F(j w)+F^{*}(j w) \geq 0
$$

where $F(j w)$ is either the admittance or impedance transfer function and * represents the complex conjugated. The family of PR transfer functions presents interesting stability properties when considered inside of a feedback loop. Among them, they are known to behave in a stable way when controlled with a RFS [22]. However, even in this convenient situation, the fact that the controlled variable is the converter-side current and not the actual grid injected current generates some undesirable side effects such as uncontrolled oscillations, un- 


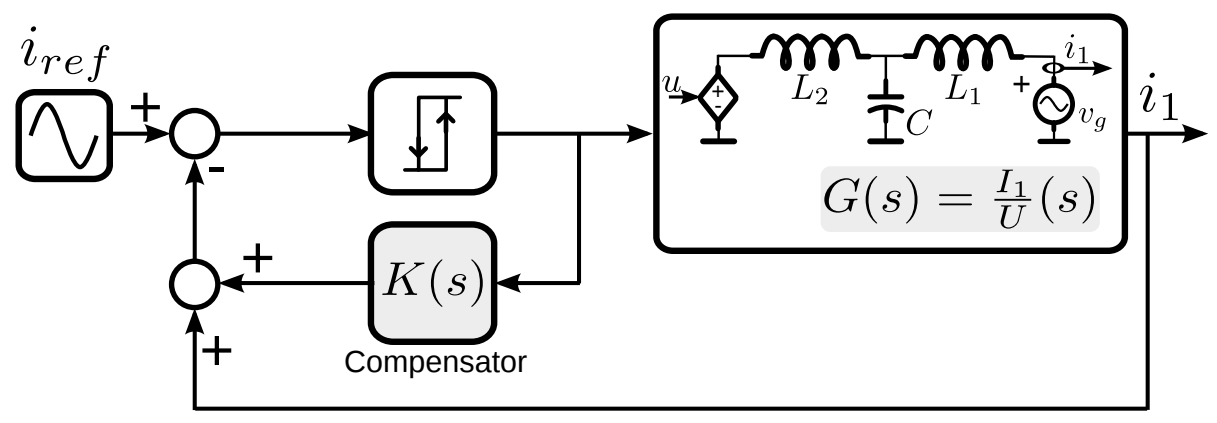

Figure 1: Hysteretic relay feedback servo system with the proposed compensator.

controlled displacement power factor (DPF) and, in general, a certain lack of control due to the partial open-loop configuration. Some partial solutions to these problems can be found in the literature [3]. Unfortunately PR properties vanish when the fed back signal is the grid-side current as the transfer function under control is not an admittance any more. A RFS-based control loop of this kind of plant is unstable.

This paper proposes the use of a compensator $K(s)$ locally feeding back the relay element as shown in Fig. 1. The function of the compensator $K(s)$ will be to render the control loop stable while ensuring that the output current ${ }_{45} i_{1}(t)$ tracks the reference signal $i_{r e f}$. This approach will rely on the concept of parallel compensation of non-minimum phase plants [8] and on the concept of Almost Strictly Positive Real (ASPR) [18] function.

Section 2 presents the theoretical foundations of the proposal. Section 3 discusses the particular application of the described methodology to the concrete problem of LCL grid-side current control. Section 4 benchmarks the proposal in a detailed simulation environment. Finally section 5 reports the main conclusions of the proposal. 


\section{Theoretical background}

\subsection{Reference signal propagation through a relay servo system}

55 Consider a relay servo system like the one displayed on Fig. 1 for the moment without the compensator $1 \frac{1}{1} K(s)$. Assume, also, that the linear part, $G(s)$, is a low-pass LTI transfer function and that the external inputs, reference and disturbances, are much slower than the self-excited oscillations characteristic of this kind of systems. In [4] it was shown that, under these assumptions,

6o the dynamics of the system can be split into two separate subsystems: a slow subsystem and a fast subsystem.

The fast subsystem is responsible for the self-excited oscillations or periodic motions (limit-cycle) characteristic of RFS while the slow subsystem deals with the forced motions caused by the reference (see Fig. 1), the disturbances or 65 the non-zero initial conditions of some elements in the system. Note also that both systems interact so the slow dynamics depend on the fast ones [4] and vice-versa.

From the slow subsystem point of view, the system averagely behaves following the closed-loop that results from replacing the relay element by an equivalent gain $k_{e q}$. The actual output additionally presents a high frequency (self-excited) oscillation that follows the fast motion model of the feedback loop.

\subsection{Stability of the control scheme. Almost strictly positive realness concept}

Assuming that the relay element behaves as an equivalent constant $k_{e q}$ for low frequency signals, the closed loop will be stable if the plant $G(s)$, when 75 connected in feedback through that constant, is stable. In this context, the concept of Almost Strictly Positive Realness plays an interesting role.

Definition 1. A linear system $P(s)$ is said to be Almost Strictly Positive Real (ASPR) [18] if there exists a constant gain $k_{e}$, not necessarily known, such

\footnotetext{
${ }^{1}$ It is worth to remark that the whole controller is composed by the relay element and the compensator $K(s)$.
} 
that the closed loop transfer function

$$
Z(s)=\frac{P(s)}{1+k_{e} P(s)}
$$

is Strictly Positive Real.

Lemma 1. [18] Let $P(s)$ be a LTI transfer function. $P(s)$ is ASPR if:

- $\operatorname{rd}\{P(s)\}=1$.

- $P(s)$ is minimum-phase: if $z_{i}$ is a system zero, $P\left(z_{i}\right)=0$, then $\Re e\left\{z_{i}\right\}<$ 0 .

Where $r d\{\cdot\}$ represents the relative degree (denominator degree minus numerator degree) of the rational transfer function $P$.

Lemma 2. [18] If $P(s)$ is an ASPR LTI transfer function then there exists a ${ }_{85} k_{n} \in \mathbb{R}$ with $k_{n}>0$ such that $1+k P(s)$ is Hurwitz for all $k>k_{n}$.

The first lemma gives the conditions for a transfer function to be ASPR while the second one provides with a stabilization method: when controlling an ASPR function, if the relay equivalent gain $k_{e q}>k_{n}$, the closed loop will be stable. The objective of the parallel compensator $K(s)$ is, thus, to transform $G(s)$ into an ASPR plant with a low enough minimal gain $k_{n}$.

\subsection{ASPR synthesis via parallel compensation}

Unlike other control schemes, as for example linear controllers, the topology of a relay feedback control scheme limits to a big degree the possible locations of user-defined transfer functions that could modify the loop behaviour or stability.

95 A traditional strategy is the use of a local relay feedback function, as shown in Fig. 1. In this scheme the compensator, $K(s)$, uses the output of the relay element, linearly modifies it and feeds it back into the relay element input again.

From the point of view of the system stability, this schema is topologically equivalent to applying a linear parallel compensator to the transfer function 


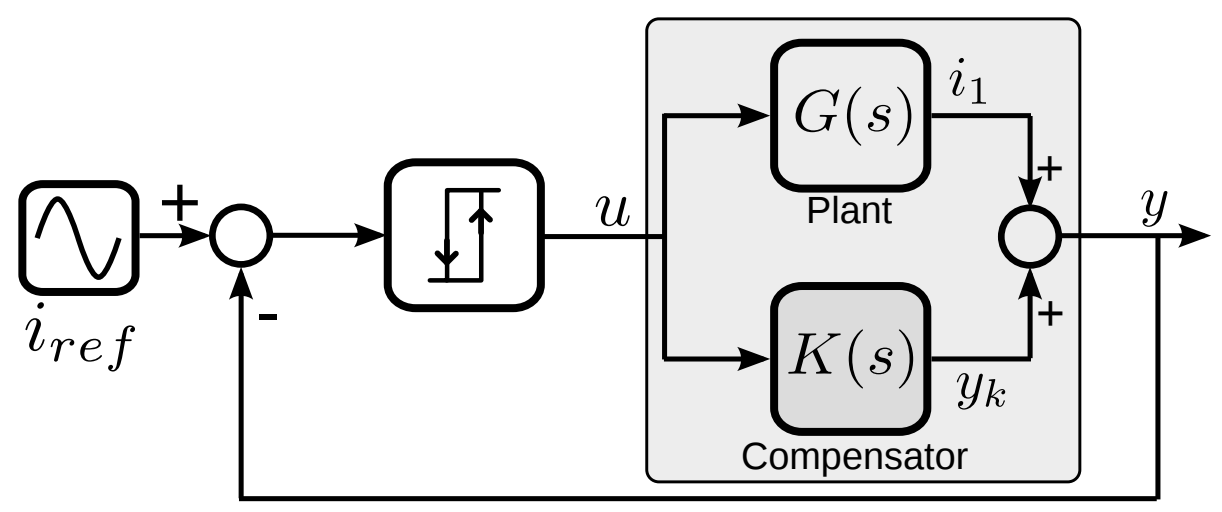

Figure 2: Compensated plant equivalent from stability point of view. in 8 .

Consider a strictly proper transfer function $G(s)$ with $r d\{G(s)\}=\operatorname{deg}\left\{d_{G}\right\}-$ $\operatorname{deg}\left\{n_{G}\right\} \geq 1$, where $n_{G}$ and $d_{G}$ stand for the numerator and denominator of $G$, respectively. Consider also the transfer functions $A(s)=\frac{n_{A}(s)}{d_{A}(s)}$ and $B(s)=$ 


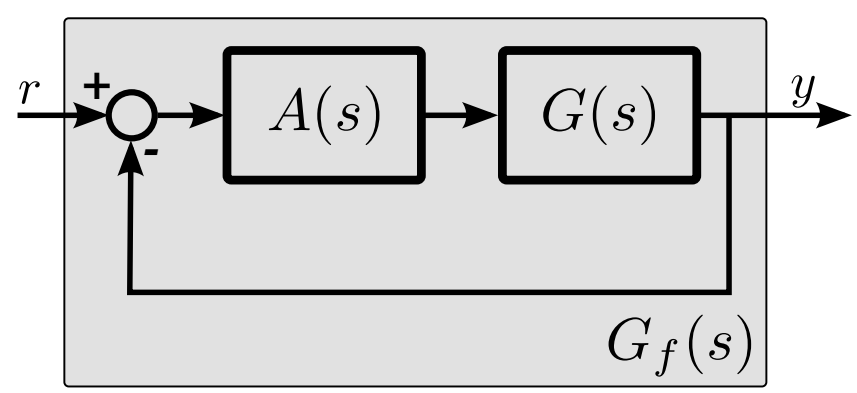

(a)

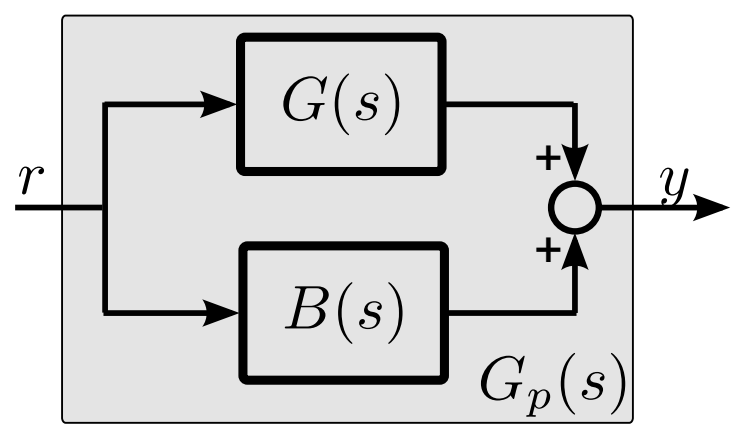

(b)

Figure 3: Negative feedback vs Parallel feedforward.

$\frac{n_{B}(s)}{d_{B}(s)}$. The transfer function relating the reference input $r$ to the output $y$ in the negative feedback scenario (Fig. 3. a) is:

$$
G_{f}(s) \triangleq \frac{Y(s)}{R(s)}=\frac{A(s) G(s)}{1+A(s) G(s)}=\frac{\frac{n_{A}(s)}{d_{A}(s)} \frac{n_{G}(s)}{d_{G}(s)}}{1+\frac{n_{A}(s)}{d_{A}(s)} \frac{n_{G}(s)}{d_{G}(s)}}=\frac{n_{A} n_{G}}{d_{A} d_{G}+n_{A} n_{G}}
$$

Similarly, the equivalent transfer function relating $r$ and $y$ in the parallel feedforward scenario (Fig. 3.b) is:

$$
G_{p}(s) \triangleq G(s)+B(s)=\frac{n_{G}(s)}{d_{G}(s)}+\frac{n_{B}(s)}{d_{B}(s)}=\frac{n_{B} d_{G}+d_{B} n_{G}}{d_{B} d_{G}}
$$

If we now consider $B(s)=A^{-1}(s)$ or in other words $n_{B}=d_{A}, d_{B}=n_{A}$, it 125 can be seen from (3) and (4) that the numerator of $G_{p}$ equals the denominator of $G_{f}$. This fact is the basis of the duality: while the negative feedback process 
allows the placement of the compensated system poles, the parallel feedforward allows the placement of the zeros.

The above property gives a method to render a plant ASPR for any strictly proper plant:

1. The condition of relative degree 1 , can be achieved remembering that, in the case of parallel compensation:

$$
r d\left\{G_{p}(s)\right\}=\min \{r d\{B(s)\}, r d\{G(s)\}\}
$$

so a $r d\left\{G_{p}\right\}=1$ can be ensured if $B(s)$ has relative degree 1 . That implies $A(s)$ with $r d\{A(s)\}=-1$.

2. The condition of $G_{p}$ being minimum phase is equivalent to being its numerator Hurwitz. As the numerator of $G_{p}$ equals the denominator of $G_{f}$, the condition is equivalent to $G_{f}$ being asymptotically stable.

Additionally it is desirable to obtain a plant $G_{p}=G+B$ with well damped zeros (poles of $G_{f}$ ) and good stability margins, to guarantee stability even for not so big $k_{e q}$ (low switching frequencies or big steps on disturbances). Some more insight into the design process can be obtained by observing the transfer function relating the reference the grid injected current $i_{1}$ and its reference $i_{r e f}$ (see Fig. 1) replacing the relay element by its equivalent gain $k_{e q}$ :

$$
\frac{I_{1}}{I_{r e f}}(s)=\frac{\frac{k_{e q}}{1+k_{e q} K(s)} G(s)}{1+\frac{k_{e q}}{1+k_{e q} K(s)} G(s)}=\frac{k_{e q} G(s)}{1+k_{e q} \underbrace{(K(s)+G(s))}_{G_{p}}}
$$

Focusing on its denominator, namely $V(s)=1+k_{e q} G_{p}(s)$, its roots will define the dynamics of the closed loop response. Equaling it to 0 and recalling equations (3) and (4):

$$
\begin{aligned}
1+k_{e q}\left(\frac{n_{B} d_{G}+d_{B} n_{G}}{d_{B} d_{G}}\right) & =0 \\
d_{B} d_{G}+k_{e q}\left(n_{B} d_{G}+d_{B} n_{G}\right) & =0 \\
V(s)=k_{e q}^{-1} d_{B} d_{G}+\left(n_{B} d_{G}+d_{B} n_{G}\right) & =0
\end{aligned}
$$


now, assuming a high $k_{e q}$ and taking limits,

$$
\lim _{k_{\text {eq }} \rightarrow \infty} V(s)=0 \quad \Longleftrightarrow \quad\left(n_{B} d_{G}+d_{B} n_{G}\right)=0 .
$$

Equation (6) indicates that the final closed loop dynamics, neglecting the limit cycle oscillation, will be marked by the roots of $\left(n_{B} d_{G}+d_{B} n_{G}\right)$, which are the poles of $G_{f}$ and the zeros of $G_{p}$.

\section{LCL grid-side current control. Parallel compensator design}

The LCL transfer function relating the converter output voltage with the grid-side current is

$$
\begin{array}{r}
G(s) \triangleq \frac{I_{1}}{U}(s)= \\
\frac{E}{L_{2} L_{1} C_{1} s^{3}+\left(R_{2} L_{1} C_{1}+L_{2} R_{1} C_{1}\right) s^{2}+\left(L_{1}+R_{2} R_{1} C_{1}+L_{2}\right) s+R_{1}+R_{2}}
\end{array}
$$

where $L_{1}, R_{1}, L_{2}, R_{2}$ stand for the inductance and resistance of the grid-side and convert-side branches of the LCL filter respectively, $C_{1}$ stands for the LCL capacitor and $i_{1}$ and $u$ are the grid-side current and converter voltage output normalized to the DC bus voltage $E$ respectively ${ }^{2}$

$G(s)$ is of order three and has no zeros. As a consequence it is far from being ASPR. Note that this situation is completely different in the case of the transfer function relating the converter output voltage with the converter-side current $i_{2}$. As this transfer function is a dissipative impedance it is strictly positive real (SPR) [1] and the RFS control loop is stable for all $k_{e q}$ (easily deducible from the fact that the polar plot of this transfer function is always in the right-hand complex plane so there is no way it can encircle the -1 point).

In order to convert $G(s)$ into an ASPR plant it is necessary to compensate it with a parallel feedforward transfer function $K(s)$ that fulfills the following requirements:

\footnotetext{
${ }^{2}$ To better illustrate the design process, all the design diagrams are obtained with the parameters on Table. 1 of Section 4
} 
1. $\operatorname{rd}\{K(s)\}=1$.

2. $G_{p}=K(s)+G(s)$ minimum-phase, that is $G_{f}=\frac{K(s)^{-1} G(s)}{1+K^{-1}(s) G(s)}$ stable.

3. $K(s)$ should be small in the frequencies where the reference is expected. In this case, we are considering a VSC delivering power at the fundamental frequency of the grid so $K(j \omega)$ should have little modulus at $50 \mathrm{~Hz}\left(\omega_{1}=\right.$ $2 \pi 50 \mathrm{rad} \mathrm{s}^{-1}$ ) to ensure $i_{1}$ is close to $y$ in Fig. 2 .

As an example design, this section considers a parallel compensator of the form:

$$
K(s)=C \frac{s+a}{\left(s+\left(b_{1}+j b_{2}\right)\right)\left(s+\left(b_{1}-j b_{2}\right)\right)}
$$

$K(s)$ fulfills relative degree requirement. The selection of the parameters $C$, $a, b_{1}$ and $b_{2}$ must ensure that new zeros stay in the left-hand complex plane so the compensated plant is minimum-phase. Fig. 4 a shows the root locus diagram of the negative feedback dual problem, $G(s)$ in negative feedback with $K^{-1}(s)$. Closed-loop poles (compensated-plant zeros) are chosen to be stable, and as damped as possible. The third requirement states the convenience of choosing a $K(s)$ with small gain in the fundamental frequency, $\omega_{1}$, in order to avoid tracking errors in $i_{1}$. Depending on the concrete application this could be obtained directly by adequately choosing $a, b_{1}$ and $b_{2}$.

If that were not enough, it is important to remark that inside the slow signal propagation domain the full LTI theory is available to ensure tracking/disturbance rejection at one or several frequencies. In this case, to ensure a good fundamental frequency tracking accuracy, a notch filter has been added to the compensator function, getting:

$$
K(s)=C \frac{s+a}{\left(s+b_{1}+j b_{2}\right)\left(s+b_{1}-j b_{2}\right)} \cdot \frac{s^{2}+2 \sigma s+\left(\sigma^{2}+\omega_{1}^{2}\right)}{s^{2}+\omega_{1}^{2}}
$$

This procedure can be extended to other typical specifications as, for example, the improvement of the attenuation of a certain grid voltage harmonic or 


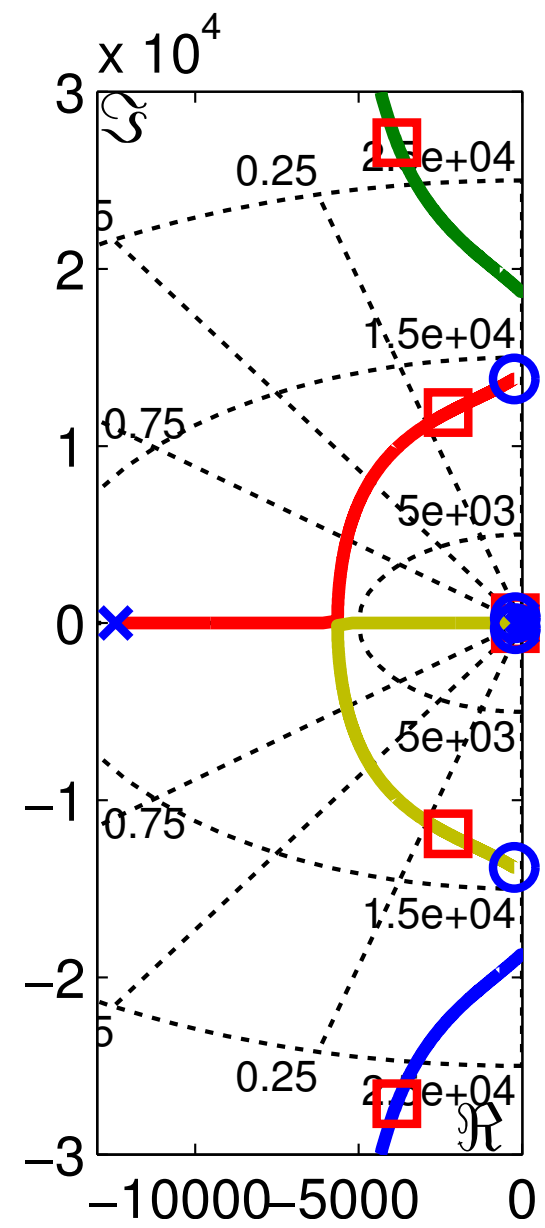

(a)

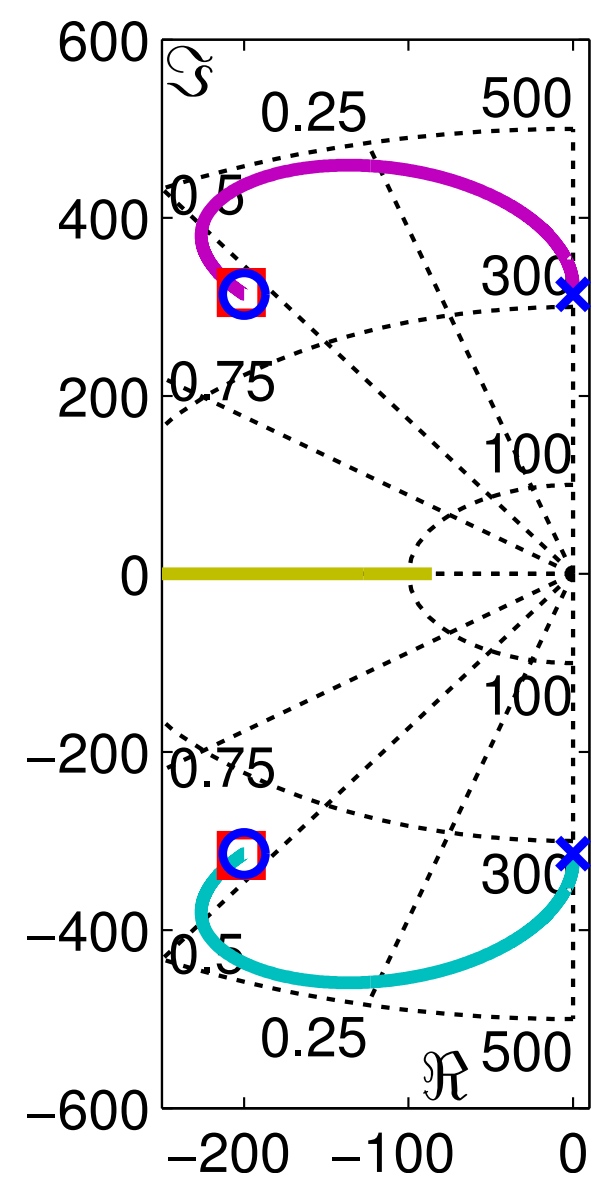

(b)

Figure 4: a. Root locus diagram of the negative feedback dual problem. The circles represent the zeros of $A$ (the poles of $K$ ), the crosses represent the poles $A=K^{-} 1$ and the squares the poles of the closed loop system $G_{f}$, that will be the zeros of $G_{p}=G+K$. b. Detail of the effect of the notch filter around $w_{1}$ over the root locus diagram. 

of the relay from minus to plus (defined at zero time), that is $-b$.

Once the LPRS of a given system is computed, the frequency of the possible limit cycle and the corresponding relay element equivalent gain $k_{e q}$ can be extracted by calculation the intersection of $J(\omega)$ with an horizontal line, which 215

the tracking of higher order harmonics, as required in active filtering applications. It has to be remarked, however, that most typical grid disturbances can alternatively be attenuated by a proper feedforward of the grid PCC voltages. This procedure is described in detail in subsection 3.3

Fig. 4 b shows a detail of the root locus branches generated with the introduction of the notch filter. Fig. 5 shows the Bode plot of the original plant $G(s)$, the parallel compensator $K(s)$ and the compensated plant $G_{p}(s)=K(s)+G(s)$. Fig. 6a shows the Nyquist plot for function $G_{p}$ and Fig 6 zooms this plot around the -1 . point. This latter subplot shows that the compensated plant polar plot crosses again the negative real axis at lower values than -1 , ensuring the -1 point is encircled twice: one in clockwise direction (marked with $(+)$ in the figure), and one in counterclockwise direction (marked with (-) in the figure). As the open loop plant does not have right-hand-plane pole (is stable), the closed-loop system will be stable for $k_{e q}>k_{0}$, being $k_{0}$ the minimum relay equivalent gain that ensures system stability.

\subsection{Frequency-domain analysis. Locus of the perturbed relay system.}

The locus of the pertubed relay system 4] (LPRS) provides the designer with an exact and usefull hodograph-based graphical analysis -and synthesis tool. It is defined as follows:

Definition 2. The Locus of a perturbed relay system is defined as the function $J(\omega):$

$$
J(\omega)=-\frac{1}{2} \frac{1}{k_{e q}}+\left.j \frac{\pi}{4 c} y(t)\right|_{t=0}
$$

where $k_{e q}$ represents the relay element equivalent gain, $c$ stands for the relay element positive output vale and $\left.y(t)\right|_{t=0}$ represents the condition of the switch

lies $\pi b / 4 c$ below $(b>0)$ or above $(b<0)$ the horizontal axis. 

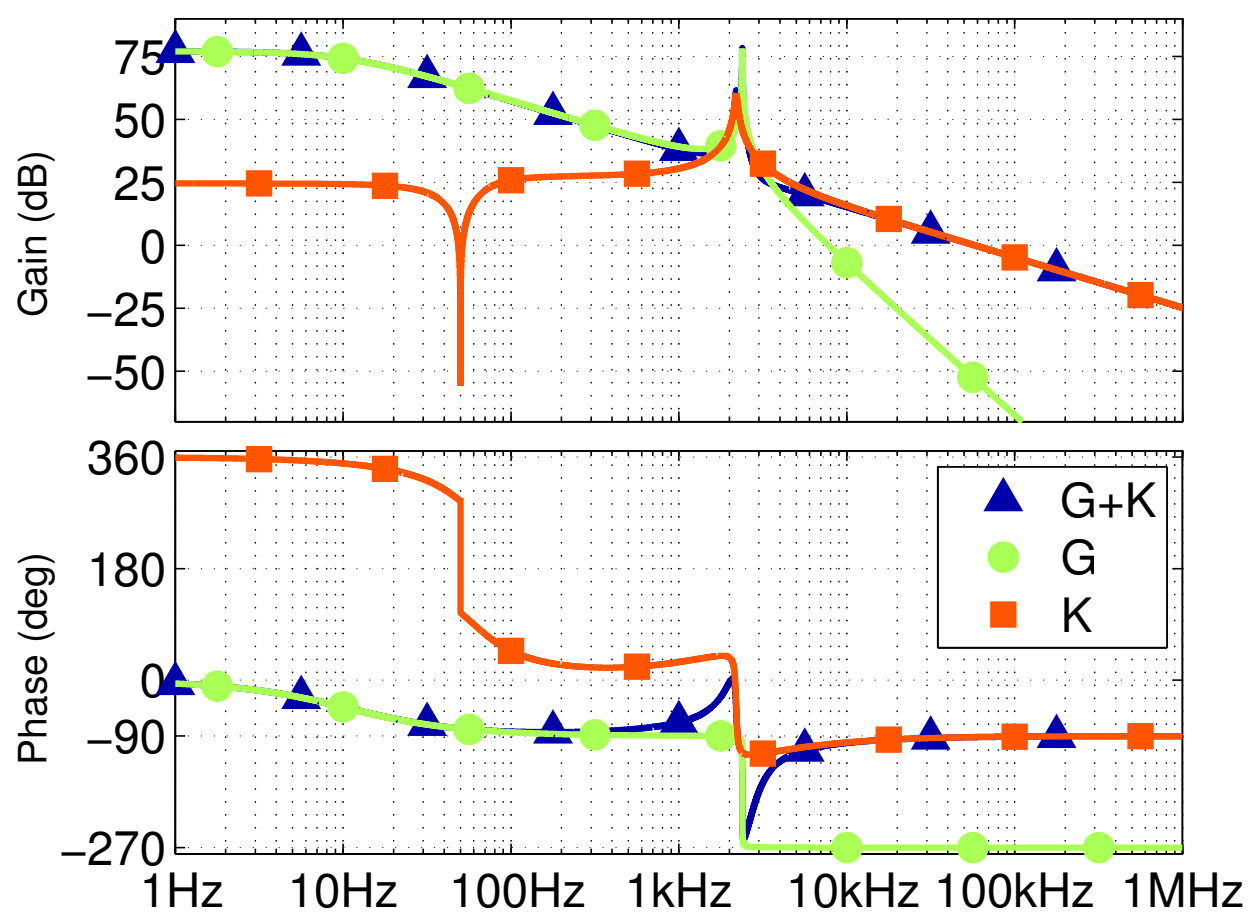

Figure 5: Bode diagram of the original plant $G(s)$, the parallel compensator $K(s)$ and the compensated plant $G_{p}(s)=K(s)+G(s)$. Top diagram represents the transfer functions modulus in dB. Bottom diagram represents their phases in degrees. 


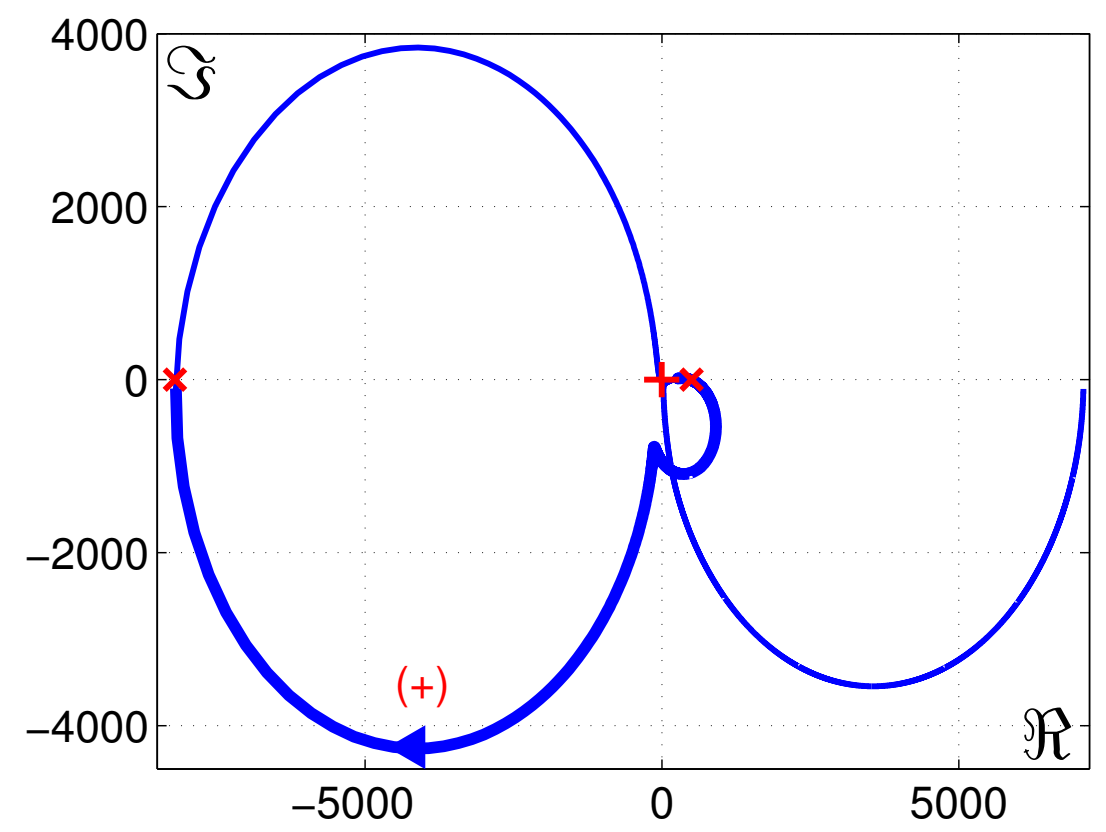

(a) Nyquist diagram of $G_{p}$.

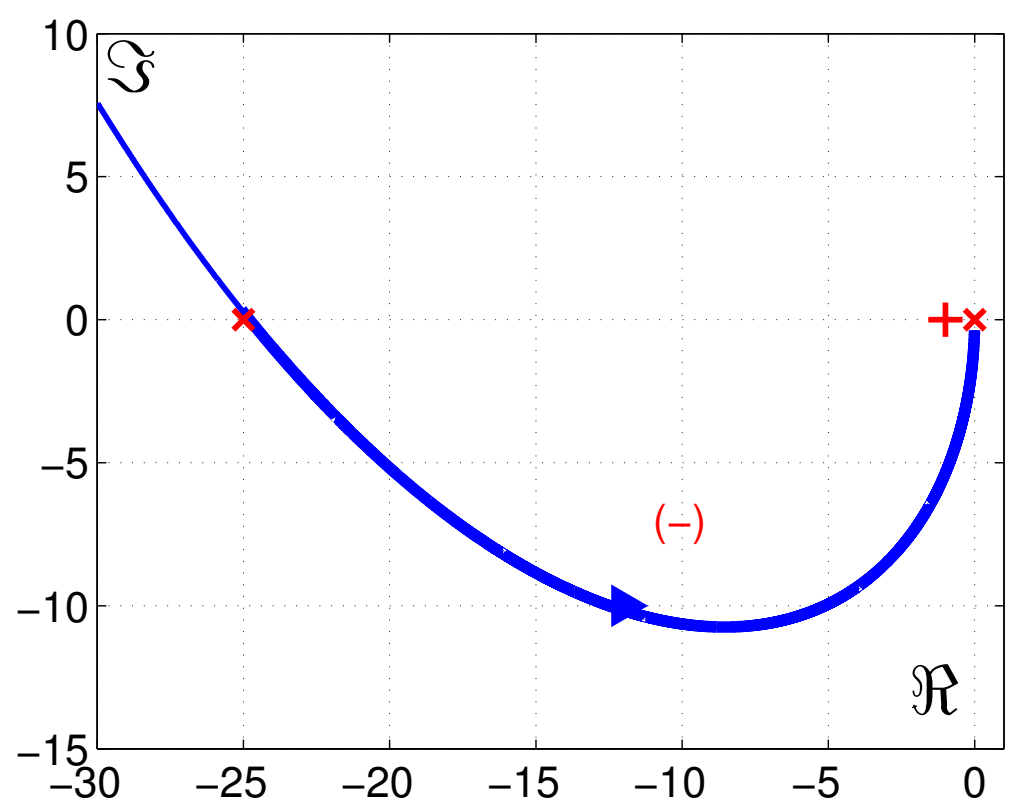

(b) Detail around -1 point.

Figure 6: Nyquist plot of $G_{p}$. 
The computation of the LPRS is extracted from the solution of the Poincaré map of the relay system:

$$
\left\{\begin{array}{l}
\eta=e^{A \theta_{1}} \rho+A^{-1}\left(s^{A \theta_{1}}-I\right) B \\
\rho=e^{A \theta_{2}} \eta+A^{-1}\left(s^{A \theta_{2}}-I\right) B
\end{array}\right.
$$

where $\rho=x(0)=x(T), \eta=x\left(\theta_{1}\right), \theta_{1}$ and $\theta_{2}$ are the positive and negative duration of the control signal $u(t)$.

For the case of a non-integrating delay-free plant, as the one here studied, the LPRS has the following explicit solution [4]:

$J(\omega)=-0.5 C\left[A^{-1}+\frac{2 \pi}{\omega}\left(I-e^{\frac{2 \pi}{\omega}}\right)^{-1} e^{\frac{\pi}{\omega} A}\right] B+j \frac{\pi}{4} C\left(I+e^{\frac{\pi}{\omega} A}\right)^{-1}\left(I-e^{\frac{\pi}{\omega} A}\right) A^{-1} B$

Fig. 7 presents the LPRS hodograph for the compensated plant $G+K$. It can be seen that, for the relay configuration above described and a relay band amplitude $c=5$, the hodograph predicts a limit cycle of $\Omega=2 \pi 1.806 \mathrm{rad} \mathrm{s}^{-1}$. The real part of the hodograph $(\Re e\{J(\omega)\})$ gives an equivalent gain $k_{e q}=1.831$. In section 4 it will be shown that this prediction is accurate.

It has to be remarked, however, that the predicted frequency of the limit cycle is valid in the case of symmetric limit cycle oscillation.

\subsection{Orbital stability}

The following theorem, presented in [2], gives necessary an sufficient condition for a relay feeback system orbit to be stable. It constitutes, then, a strong condition for the local stability of the relay feedback compensated system presented in this proposal.

Theorem 3. 2] The relay feedback system shown in Fig 1 is locally orbitally stable if and only if all eigenvalues of the matrix:

$$
\Phi_{O}=\left[I-\frac{v\left(\frac{T}{2}-\right) C_{C}}{C_{C} v\left(\frac{T}{2}-\right)}\right] e^{A_{C} \frac{T}{2}},
$$


where $T=\frac{2 \pi}{\Omega}$ is the period of the oscillations, $C_{C}$ and $A_{C}$ are the $C$ and $A$ matrix of the compensated plant $G+K$, respectively, and $v=\dot{x}$ is the velocity matrix,

$$
v\left(\frac{T}{2}-\right)=2\left(I+e^{A \frac{T}{2}}\right)^{-1} e^{A \frac{T}{2}} B,
$$

have magnitudes less than one (yellow trace) in Fig. 7. It can be seen that, according to the described theory, there exists a maximum relay bandwidth ( $b_{\text {limit }}$ value) that gives the minimum $k_{e q}$ that generates a stable limit cycle in the system. For $b<b_{\text {limit }}$, the $k_{e q}$ is bigger and the limit cycle would be always stable.

245

In this case, the minimum frequency for a stable orbit is $3.97 \mathrm{kHz}$. This value is very close to the falling edge of the LCL resonance (see Fig. 5). So, in the same way that happens in PI control approaches and in hysteresis control of the bridge-side current [6], the LCL resonance practically limits the minimum system switching frequency. As this parameter strongly influences system losses, a complete design may include, and in practice indeed usually does, not only the controller, but also the filter shaping.

Regarding the robustness with respect to plant parameters, it is difficult to extract conclusions from 12 due to its inherent complexity. Design experience says that a major objective for particularly robust designs is to create a compenThis is an LTI design problem that may be faced up with classical procedures. Once the plant is ASPR for the desired conditions, the limit values for stable orbit periods can be easily obtained with the aforementioned tools, just as in the Fig. 7 case.

\subsection{Disturbance feedforward compensator}

One of the advantages of Boiko's LPRS-based analysis is that it gives the designer the possibility of applying linear compensators to improve performance 


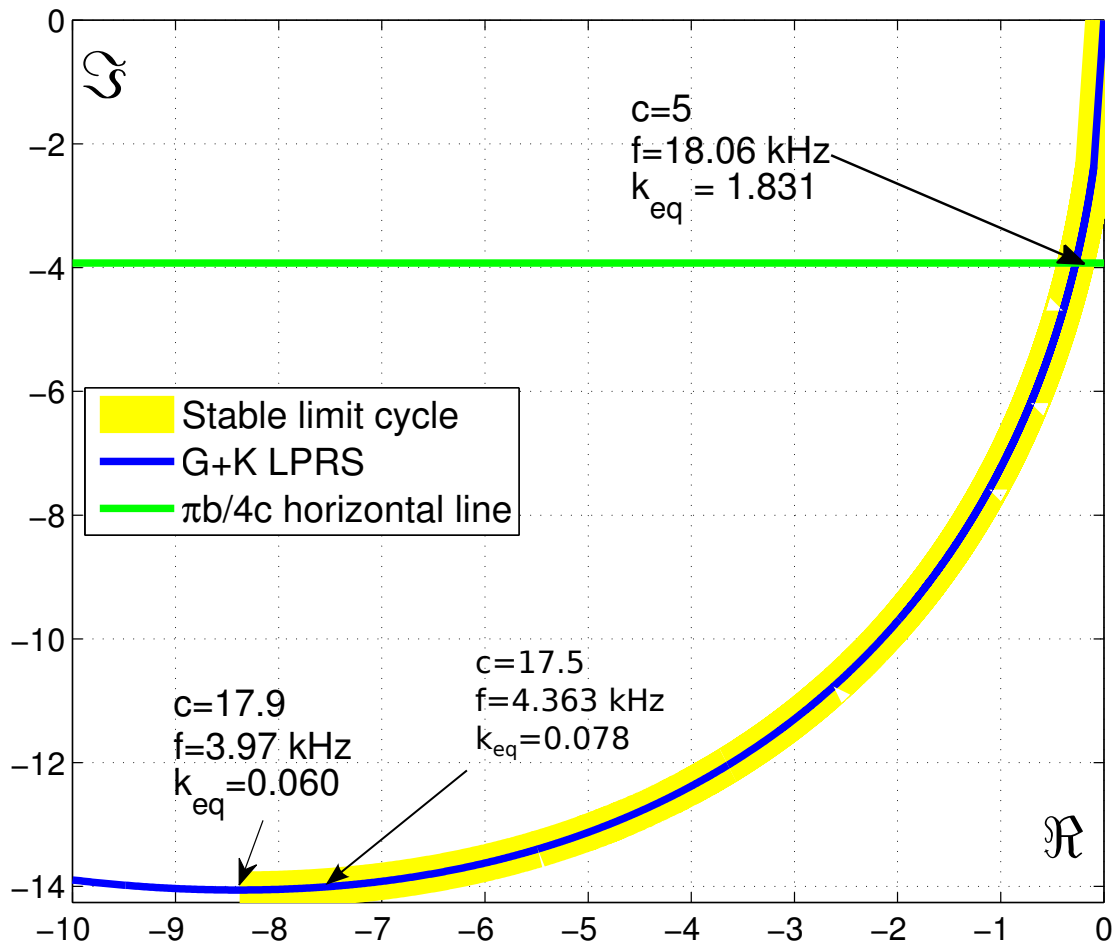

Figure 7: Locus of the perturbed relay system hodograph for the compensated plant $G+K$. The intersection point between $J(\omega)$ and the $\frac{-\pi b}{4 c}$ is marked. The thin blue trace shows $J(\omega)$. The thick yellow trace shows the frequencies whose potential limit cycle would be stable for the compensated plant. 
by substituting the relay element by its equivalent gain $k_{e q}$, if this improvement is limited to the slow-dynamics of the relay feedback system.

In LCL grid-connected applications, it is sometimes preferred to compensate the influence of grid voltage disturbance. This compensation eliminates some tracking and transient issues at very small cost: grid voltage is always sensed for synchronisation purposes or higher hierarchy loops, so only the compensator complexity is lightly increased. It provides a way, for example, to improve current tracking at fundamental frequency when voltage grid harmonics are present.

Fig. 8 $\mathrm{b}$ shows the proposed solution. It consists in the addition of the transfer function $K_{d}(s)$ output to the previous compensator $K$. The input to $K_{d}$ is the grid measured signal $v_{g}$. For design purposes, the LCL behaviour has 275 been split into the bridge output voltage-to-output current $G$ transfer function, and the grid voltage-to-output current $G_{d}$ transfer function.

The system operation in the presence of such a compensator is described by the following equations:

$$
\begin{aligned}
i_{1} & =\frac{k_{e q} G}{1+k_{e q}(G+K)} \cdot i_{\text {ref }}+\frac{-K_{d} G k_{e q} K+G_{d}\left(1+k_{e q} K\right)}{1+k_{e q}(G+K)} \cdot v_{g} \\
e & =\frac{1+k_{e q} K_{d}}{1+k_{e q}(G+K)} \cdot i_{r e f}-\frac{-K_{d} G k_{e q} K+G_{d}\left(1+k_{e q} K\right)}{1+k_{e q}(G+K)} \cdot v_{g}
\end{aligned}
$$

To obtain a good compensation of the disturbance, the effect of $v_{g}$ signal over the error signal should be null. Imposing that condition over (14):

$$
-K_{d} G k_{e q} K+G_{d}\left(1+k_{e q} K\right)=0
$$

The theoretical expression for the compensator $K_{d}$ is the following:

$$
K_{d}=\frac{G_{d}}{G} \cdot \frac{1+k_{e q} K}{k_{e q} K}
$$

This $K_{d}$ expression is, for the case under study, acausal. That is due to the fact that $G$ and $G_{d}$ present relative degree 3 and 1, respectively. Both 
transfer functions share the same denominator, so the result of the quotient is $G_{d}$ numerator. To partially solve it, two high frequency poles with unity de gain are added to the compensator. The poles frequencies should be well above the system resonance to avoid modifying the fundamental compensator behaviour:

$$
K_{d}=\frac{G_{d}}{G} \cdot \frac{1+k_{e q} K}{k_{e q} K} \cdot \frac{p}{s+p}, \quad|p|>\sqrt{\frac{1}{L_{2} C}}
$$

Fig. 9 shows the Bode plot of the perfect (acausal) disturbance compensator and the proposed causal approximation, following 16 .

\section{Simulation results}

The proposed design has been tested under a Matlab/SimPowerSystems simulated model. Table 1 summarises the main parameters of the simulated system.

Fig. 10 shows the response of the system in the presence of a voltage dip in the grid. During the grid fault, the voltage magnitude decreases to a $30 \%$ and its phase jumps $\pi$ rad. It can be seen that the current quickly recovers its reference. There is a short transient oscillating at the frequency of the $G_{p}$ complex-conjugated zeros. The transient dissapears quickly, adding a negligible impact over the main quality index as, for example, THD. Additionally, when dealing with LCL connection, it is quite common to find that the oscillations during the falling edge of a dip are less present in an experimental setup than in the simulations. The non-infinite falling slope of the grid voltage and the nonmodelled resistive losses usually add some extra damping, improving system response.

Fig. 11 shows the response of the current controller under a step reference in both magnitude and phase of the grid injected current reference $\left(i_{1}\right)$. Again the grid-injected current quick and accurately tracks the imposed reference. Fig. 12 makes a zoom on the evolution of the controlled system during the second setpoint change in Fig. 11. Top plot of Fig. 12 shows the relay hysteresis band, the compensated plant $G_{p}$ output, showing the characteristic limit cycle, and 


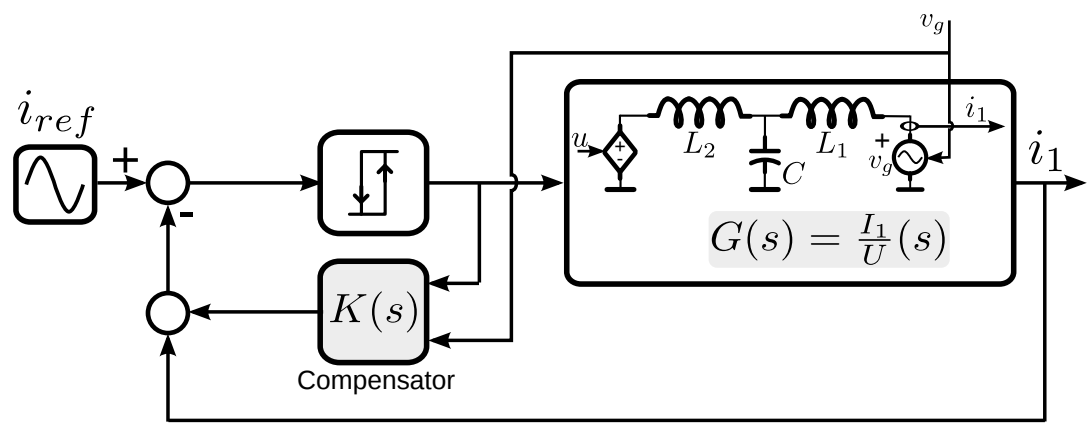

(a) Addition of the grid disturbance information into the compensator.

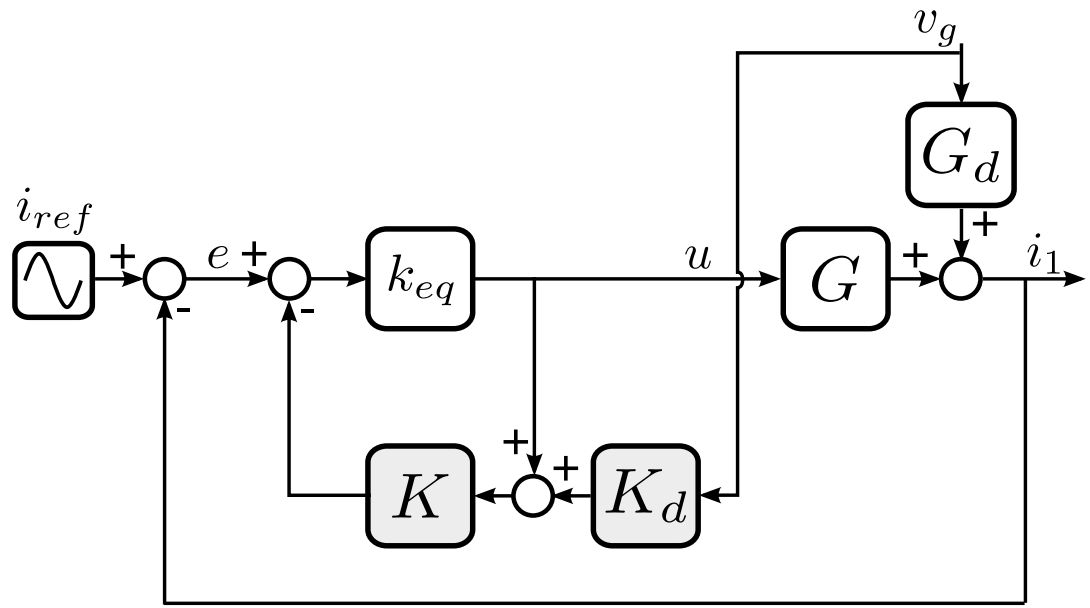

(b) Block diagram of the proposed solution for disturbance compensation. $G$ and $G_{d}$ represent the transfer functions relating the grid output current $i_{1}$ with the plant input $u$ and plant disturbance $v_{g}$ respectively. $K_{d}$ is the transfer function of the disturbance compensator. $k_{e q}$ is the relay equivalent gain obtained by means of the LPRS

Figure 8: Disturbance feedforward compensator 
Table 1: Simulation parameters

\begin{tabular}{|c|c|c|}
\hline Simulation step & $t_{\text {sim }}$ & $0.2 \mu \mathrm{s}$ \\
\hline Converter nom. pow. & $P_{n}$ & $8 \mathrm{kV} \mathrm{A}$ \\
\hline DC bus nominal voltage & $V_{D C}$ & $850 \mathrm{~V}$ \\
\hline LCL grid-side inductor & $L_{1}, R_{1}$ & $0.2 \mathrm{mH}, 10 \mathrm{~m} \Omega$ \\
\hline LCL converter-side ind. & $L_{2}, R_{2}$ & $0.5 \mathrm{mH}, 50 \mathrm{~m} \Omega$ \\
\hline LCL capacitor & $C$ & $20 \mu \mathrm{F}$ \\
\hline Grid nom. voltage & $V_{g}$ & $230 \mathrm{~V}$ \\
\hline Grid nom. fundamental freq. & $\mathrm{f}$ & $50 \mathrm{~Hz}$ \\
\hline Grid line base inductance & $L_{g}$ & $0.1 \mathrm{mH}$ \\
\hline Hysteresis band half-width & $c$ & 5 \\
\hline \multirow[t]{6}{*}{$K(s)$ parameters } & $C$ & 16.91 \\
\hline & $a$ & -12443.67 \\
\hline & $b_{1}$ & -236.18 \\
\hline & $b_{2}$ & 13793.10 \\
\hline & $\sigma$ & -0.02 \\
\hline & $\omega_{1}$ & $2 \pi 50 \mathrm{rad} \mathrm{s}^{-1}$ \\
\hline
\end{tabular}




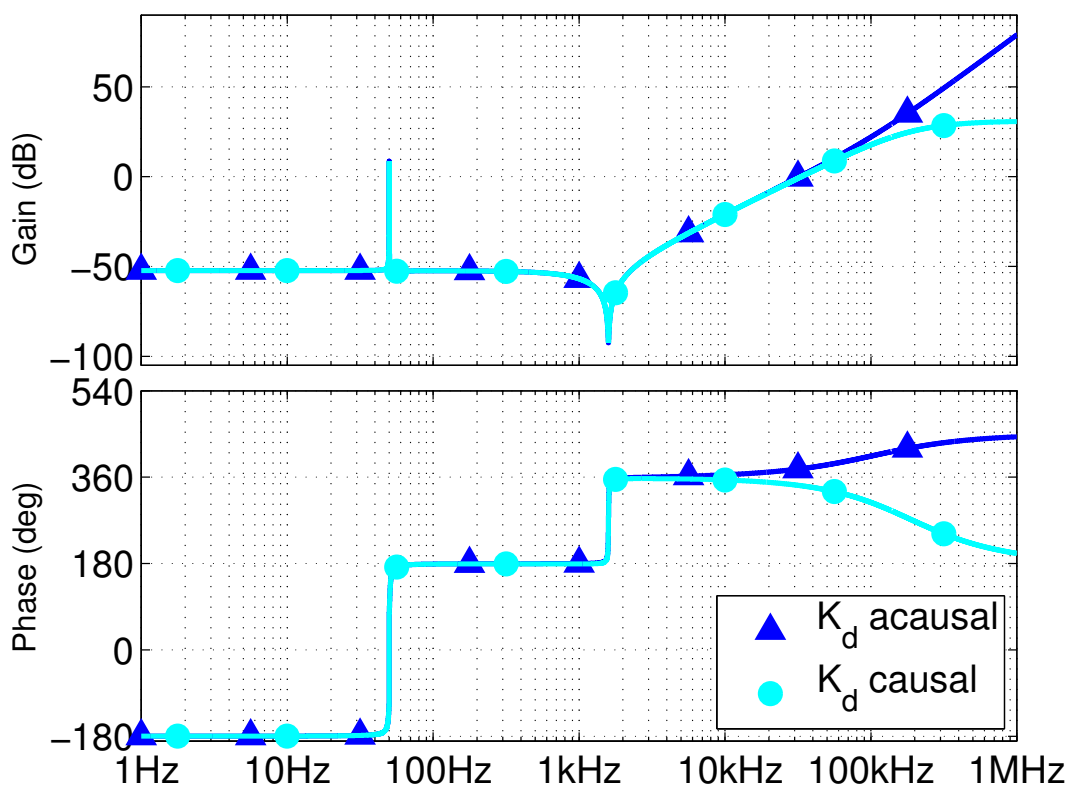

Figure 9: Disturbance feedforward compensator. Perfect (acausal) feedforward compensator in dark-blue(round marker)

the grid injected current $i_{1}$. The oscillation that is visible in $i_{1}$ is due to the complex zeros in $G_{p}$. Middle plot of Fig. 12 shows the output of the parallel compensator $K$ that is added to the output signal $i_{1}$ and introduced in the hysteretic comparator (relay) input. Bottom plot of Fig. 12 shows the output of the hysteretic comparator and input to the plant $G$.

To comply with the different existing grid codes, the converter closed loop grid voltage admittance has to attenuate the possible grid voltage harmonics. From Fig. 2 and the following development, shown in section 2 it is easy to see that if the compensator $K(s)$ presents a nonzero modulus $\left|K\left(j f_{h}\right)\right|>0$ at a frequency $f_{h}$, the grid current $i_{1}$ is going to present some difference with respect to the hysteresis controlled variable $y$. As a consequence, the grid current may have a nonzero $f_{h}$ component. That is the case of the design example described in section 3 Fig. 13 a shows the response of the system when, in $t=0.02 \mathrm{~s}$, a $30 \%$ fifth harmonic is added to grid PCC voltage. The grid current is, as expected, distorted. It can be seen that $y$ is well inside the hysteresis band, 


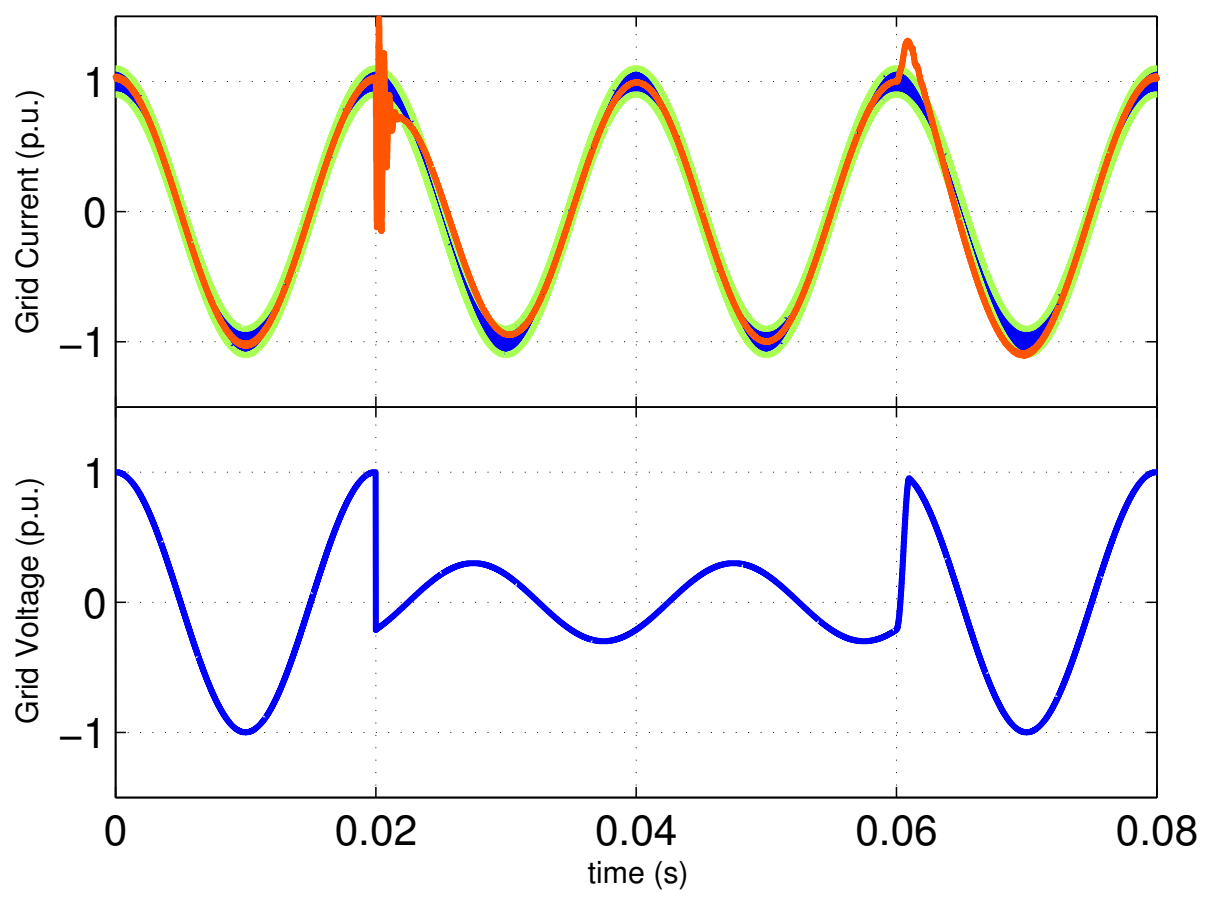

Figure 10: Current controller response during a 30\% voltage dip. Top: $i_{1}$ (red line) , $y=G_{p}(u)$ (blue line), and hysteresis bands (light green lines). Bottom: Grid voltage. 


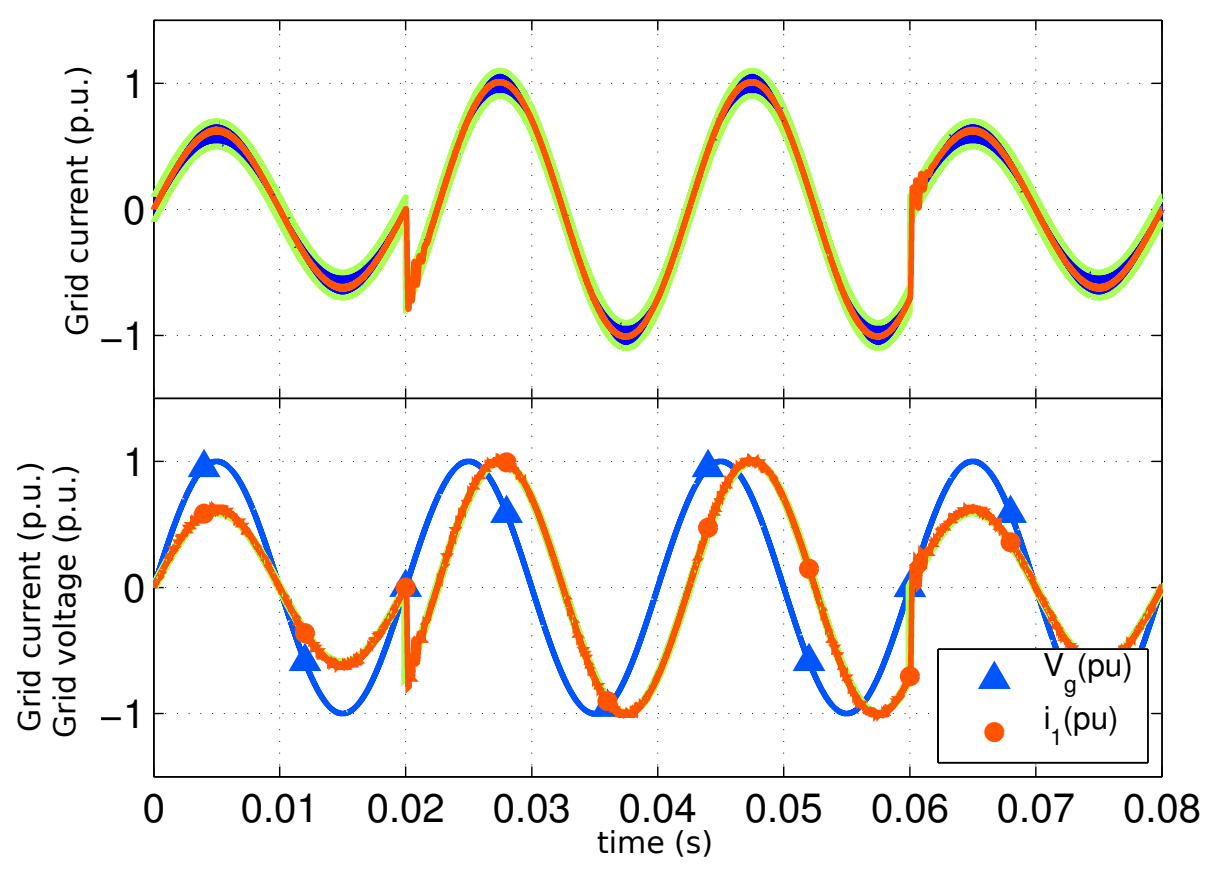

Figure 11: Current controller response under a magnitude and phase current reference step change. Top: $i_{1}$ (red line), $y=G_{p}(u)$ (blue line), and hysteresis bands (light green lines). Bottom: Grid voltage and grid injected current. 


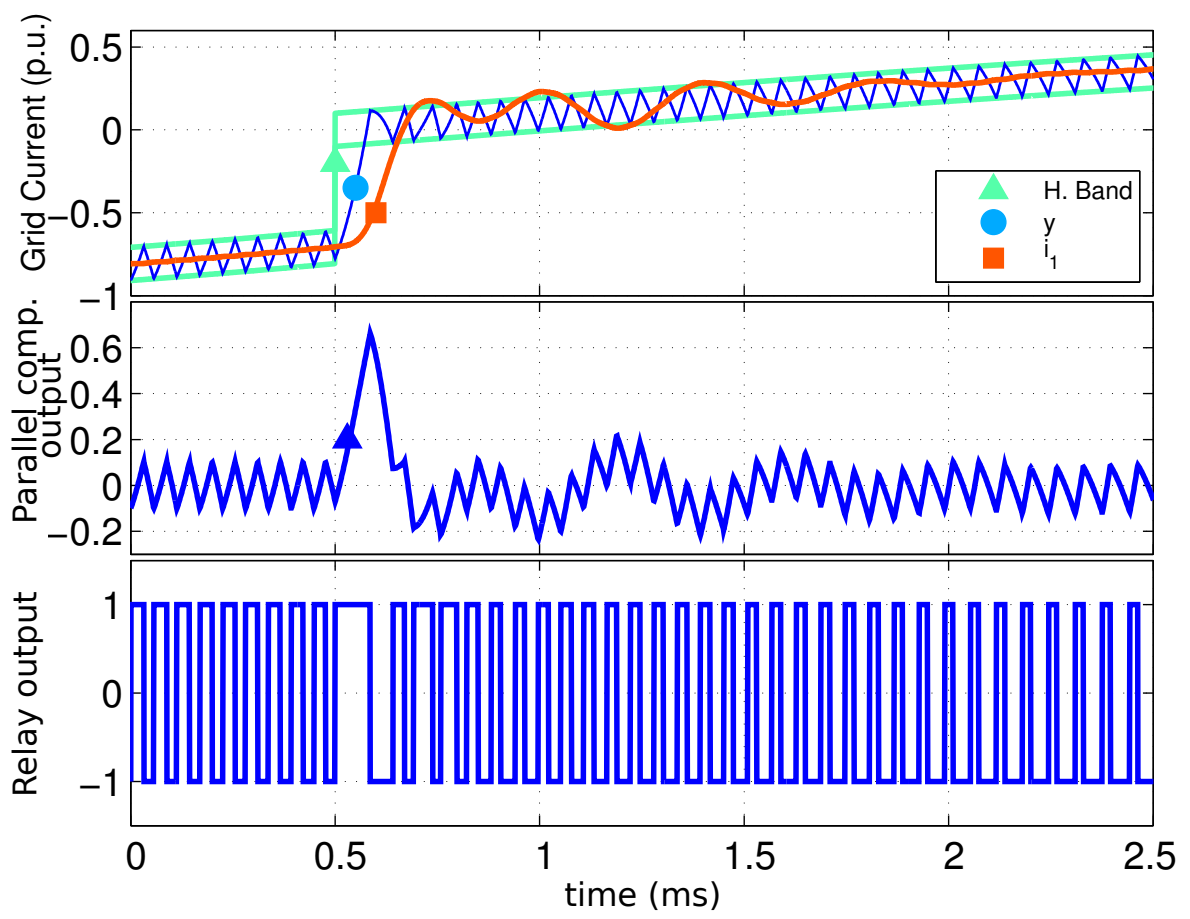

Figure 12: Detail of the response of the compensated hysteretic controlled system during a magnitude and phase current reference step change. Top: $i_{1}, y=G_{p}(u)=(G+K)(u)$ and hysteresis bands. Middle: Output of parallel compensator $K$. Bottom: Output of the hysteretic comparator and input to the plant $G$. 

of the fundamental component (fundamental component peak value: $50.95 \mathrm{~A}$, $\mathrm{THD}=8.5 \%)$.

Although the design presents some natural attenuation that could be enough for certain applications, the proposed method allows two alternative ways to improve it. The first option is to modify the parallel compensator $K(s)$ so that it presents zero/low gain at the frequency of interest. This could be done, for example, introducing another notch filter at that frequency. An alternative method is the use of the grid disturbance feedforward described on section 3.3 In fact using this feedforward allows to eliminate nearly any influence from the grid. Fig. 13b shows the effect of introducing the grid feedforward compensator at $t=0.02 s$. It can be seen that the grid current quickly decreases its distortion to $\mathrm{THD}=0.54 \%$.

Again it can be remarked that, as the hysteresis loop remains stable, the low bandwidth dynamics can be compensated with classical LTI theory, so the designer can make use of the tool of their choice to solve classical energy quality issues.

Another typical source of distortion in single-phase applications is the existence of a $2 \cdot f_{1}$ component in the DC bus energy that induces an oscillation in the bus voltage. Hysteresis controller shows a natural good behaviour in the presence of this perturbation. From a practical point of view it is important to guarantee that in the lowest voltage value the system still follows a stable orbit. An increased DC bus voltage would move the system towards higher frequency orbits where stability is guaranteed, as can be seen in Fig. 7.

To evaluate the sensitivity of the proposed current controller under the described oscillation, Fig. 14 shows the system behaviour when the DC bus voltage oscillates at twice the fundamental frequency. This approximated scenario allows to test the systems under oscillations that would appear under a broad variety of system conditions and parameters. More concretely, the system is tested under an oscillation of 10 (THD: $1.62 \%$ ), 25 (THD: $4.16 \%$ at $425 \mathrm{~V}$, $3552.85 \%$ at $600 \mathrm{~V})$, and $50 \%(\mathrm{THD}=8.52 \%$ at $600 \mathrm{~V}, 6.24 \%$ at $800 \mathrm{~V})$ of the DC 


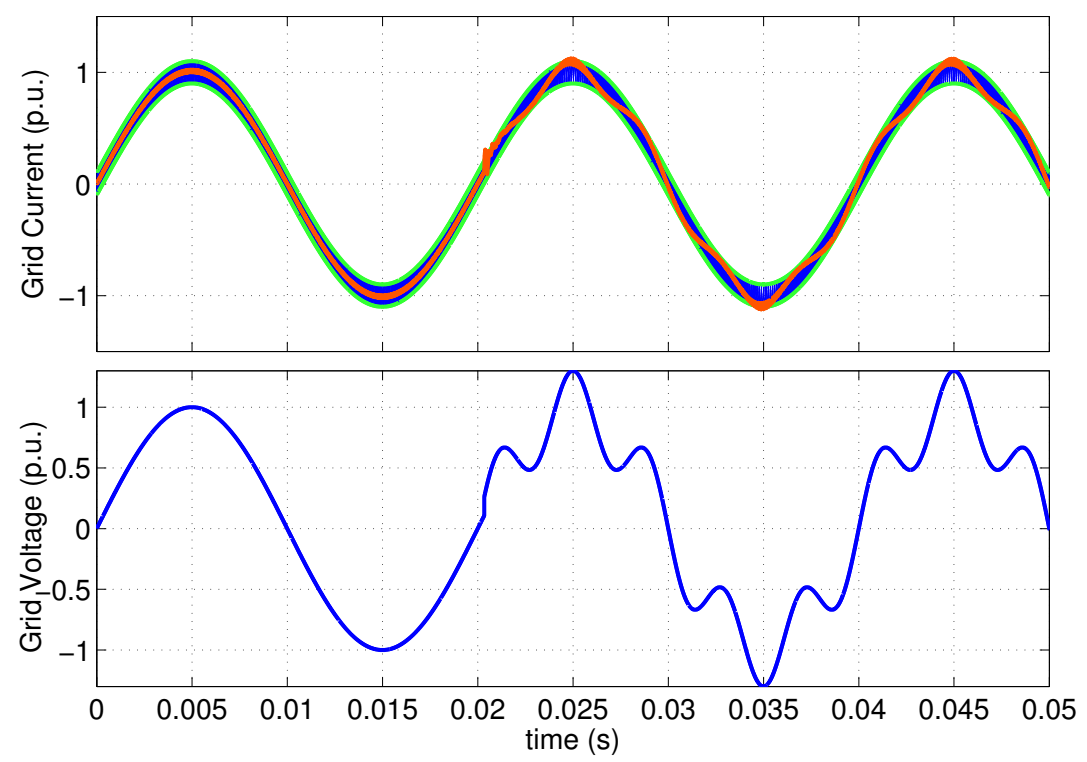

(a) Response without grid feedforward.

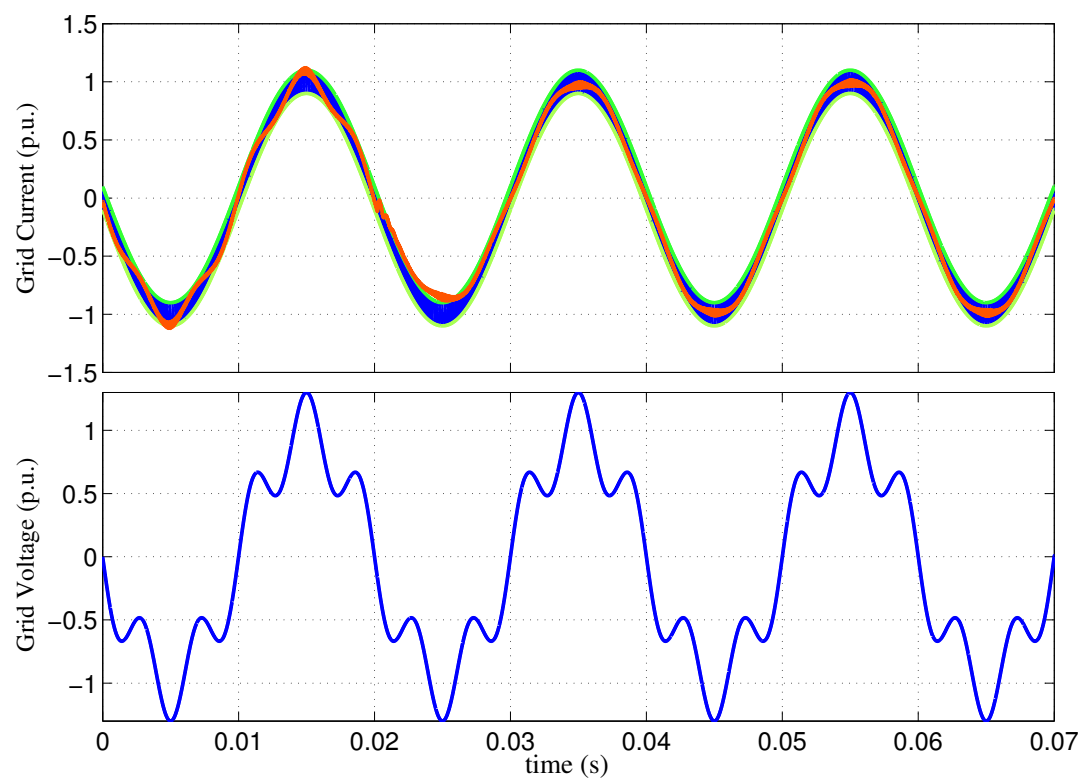

(b) Effect of grid feedforward (connected on $\mathrm{t}=0.02 \mathrm{~s}$ ).

Figure 13: Current controller behaviour under $30 \% 5^{\text {th }}$ harmonic presence. Top: $i_{1}$ (red line) , $y=G_{p}(u)$ (blue line), and hysteresis bands (light green lines). Bottom: Grid voltage. 

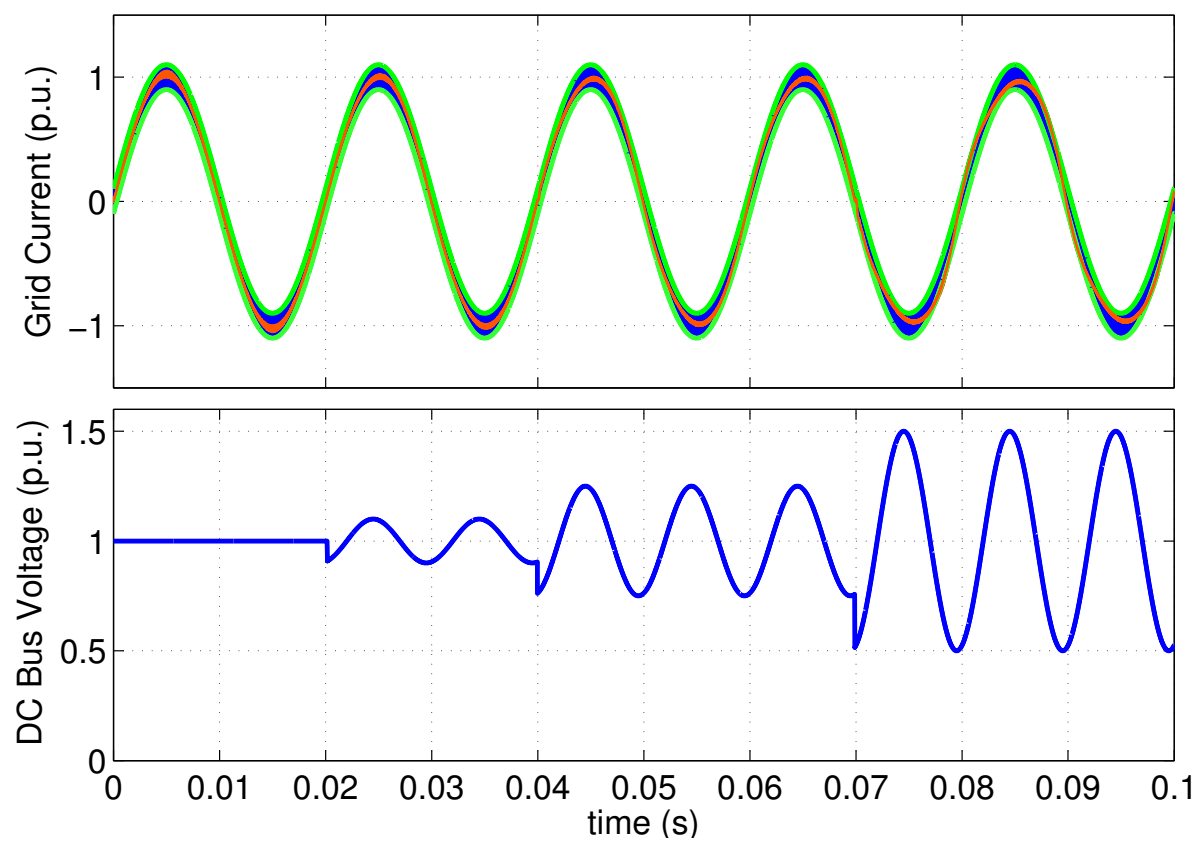

Figure 14: Current controller behaviour under a $2 f_{1}$ oscillation in the DC bus voltage of 10 , 25 and $50 \%$ of the bus nominal voltage value. Top: $i_{1}$ (red line), $y=G_{p}(u)$ (blue line), and hysteresis bands (light green lines). Bottom: DC bus voltage.

bus voltage value. The oscillation influence increases with its amplitude but is kept under acceptable levels given the DC bus conditions of the tests. It is important to see that the effect is mitigated by increasing bus voltage. Again, the $y$ signal shows a stable, periodic orbit, so some LTI compensators could be used to decrease this effect, if desired.

The presented scheme shows also a natural robust behaviour with respect to parameter variation. One parameter that always presents some uncertainty is the grid equivalent impedance, seen from the PCC.

Fig. 15 shows the behaviour of the control loop for different values of the grid equivalent inductance. It can be seen that the controller starts showing unstable behaviour for twice the grid inductance design value (100\% uncertainty). The same results are obtained if the grid equivalent inductance is not only uncertain, but time-variant, as shown on Fig. 16. It has to be noted that, in this case, the 


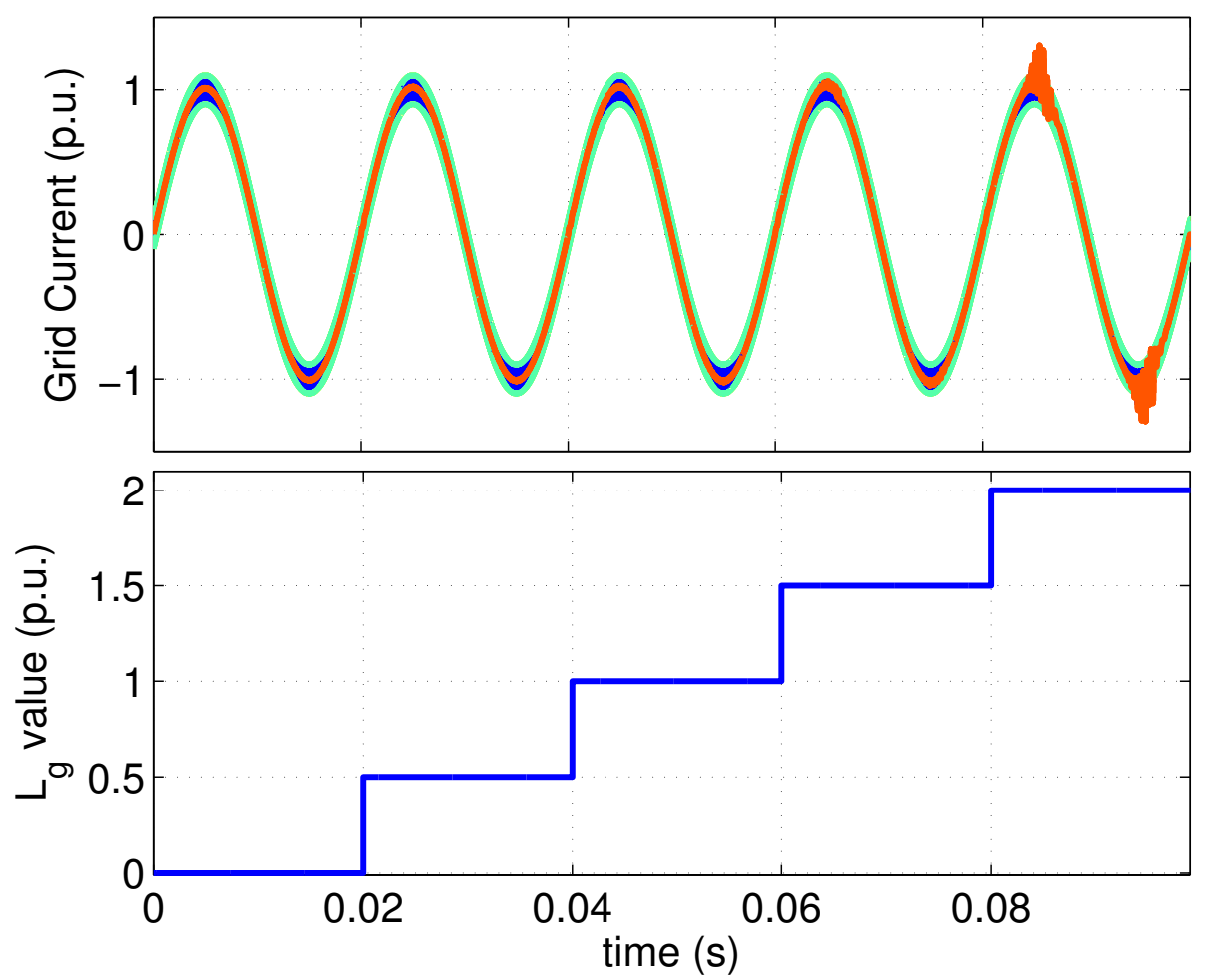

Figure 15: Current controller behaviour for different constant values of the grid equivalent inductance. Top: $i_{1}$ (red line), $y=G_{p}(u)$ (blue line), and hysteresis bands (light green lines). Bottom: Grid equivalent inductance value.

design was formulated without considering any grid uncertainty. However the ASPR synthesis procedure may be reformulated in a robust fashion using LTI theory.

Fig. 17 shows the behaviour of the system for several hysteresis band values, $c$. Fig. 17. a shows the system behaviour, switching period $\$^{3}$ and current spectrum for $c=5$. It can be seen that the switching frequency is influenced by

\footnotetext{
${ }^{3}$ The terms switching period or frequency are not strictly correct when there are perturbation or reference signals entering the loop because the limit cycle looses its periodicity. When used, they refer to the time lapsed between two consecutive switchings of the relay element and its inverse, respectively.
} 


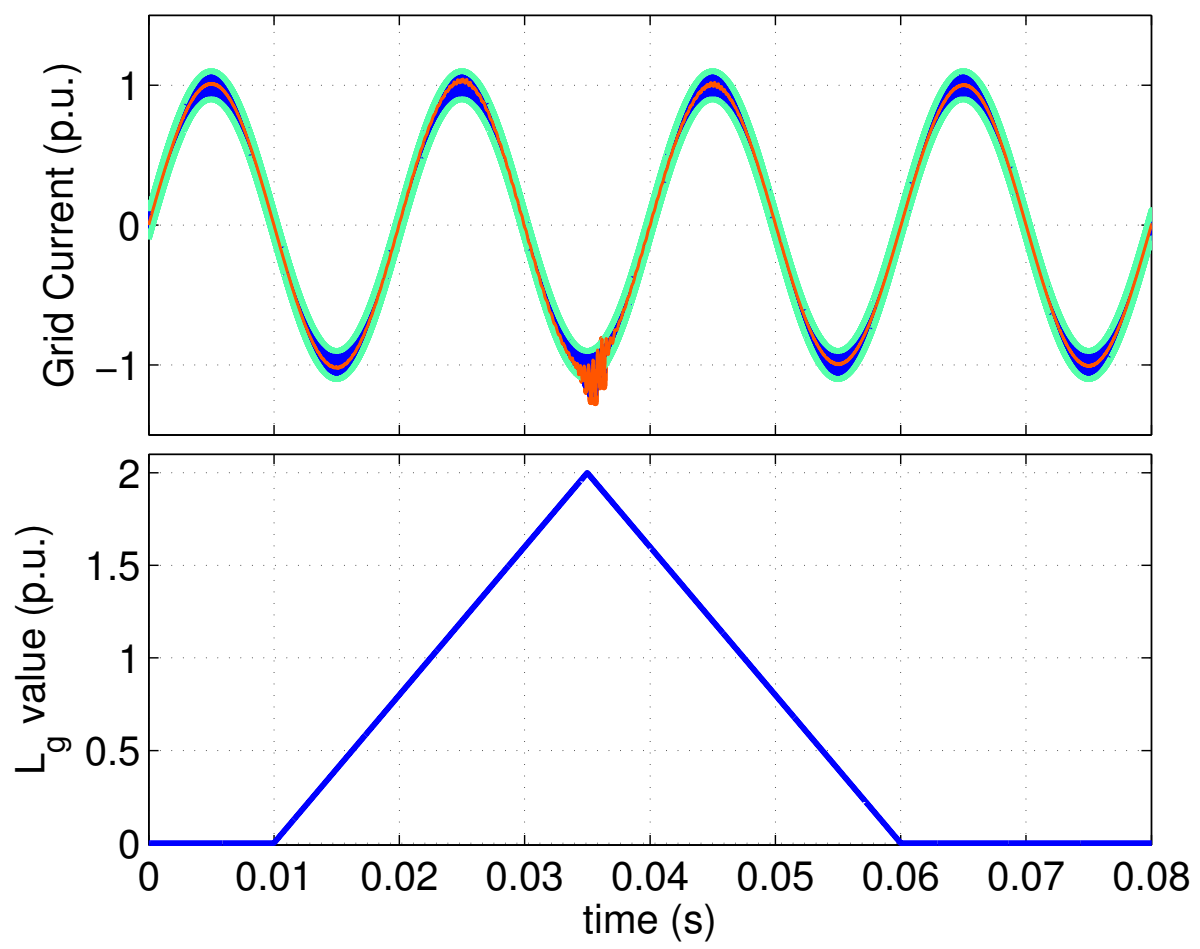

Figure 16: Current controller behaviour during a variation in the grid equivalent inductance. Top: $i_{1}$ (red line), $y=G_{p}(u)$ (blue line), and hysteresis bands (light green lines). Bottom: Grid equivalent inductance value. 
exogenous perturbation signals as the grid voltage and current reference. This is a common behaviour in relay controllers and can be mitigated using a classical variable hysteresis band [5, 11, 24] equalisation algorithm. Fig. 17. b shows the system behaviour when $c=9$. This value, when the effect of the grid over the switching frequency is considered, represents a limit stability value. Note

that the slowest switching cycles last $250 \mu \mathrm{s}$, whose corresponding frequency is beyond the limit shown in Fig. 7. Finally, Fig. 17. c shows the system behaviour for $c=17.5$ in the absence of grid voltage (short-circuit). In this case, although sinusoidal grid current reference also induces a variation on the switching frequency (see the detail shown on bottom axis of Fig 7 c), its magnitude is very small because the influence of the current reference over the control loop is much smaller than the grid voltage one.

In this case, the hysteresis band and switching period values are slightly smaller than those of the critical stability point. It can be seen that the obtained frequency slightly oscillates around the predicted one on Fig. 7.

The right column plots of Fig. 17 display the harmonic content of the injected current for the three different considered situations. As expected, the three plots show a big component on the fundamental frequency and switching distortion caused by the hysteresis induced limit cycle. In the first two cases the switching frequency is variable and, consequently, the distortion is distributed

on a frequency band. In the third case, the switching band is very narrow because of the smaller influence of the current reference on the control loop orbital behaviour. It can be seen that THD of the injected current increases as the hysteresis band increases and switching frequency decreases. This behaviour is expected because the LCL filter attenuation is smaller for lower frequencies.

\section{Conclusions}

This paper has presented a method that allows the use of a hysteretic comparator to control the grid-side current of a LCL grid-connected VSC. The method is based in the synthesis of an Almost Strictly Positive Real transfer 

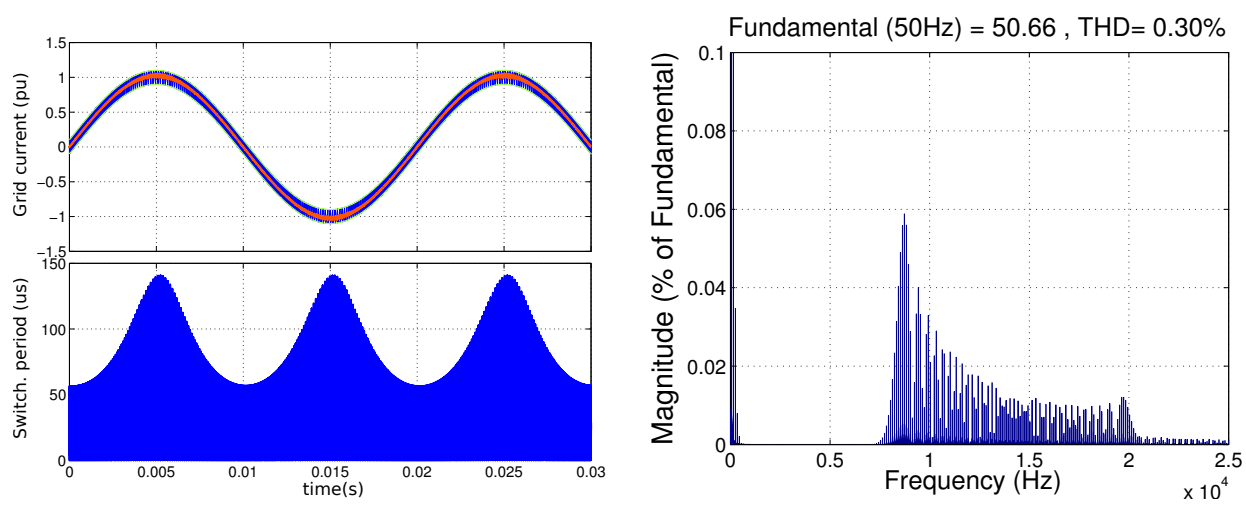

(a) $c=5 . V_{g}=220 \mathrm{~V}$.
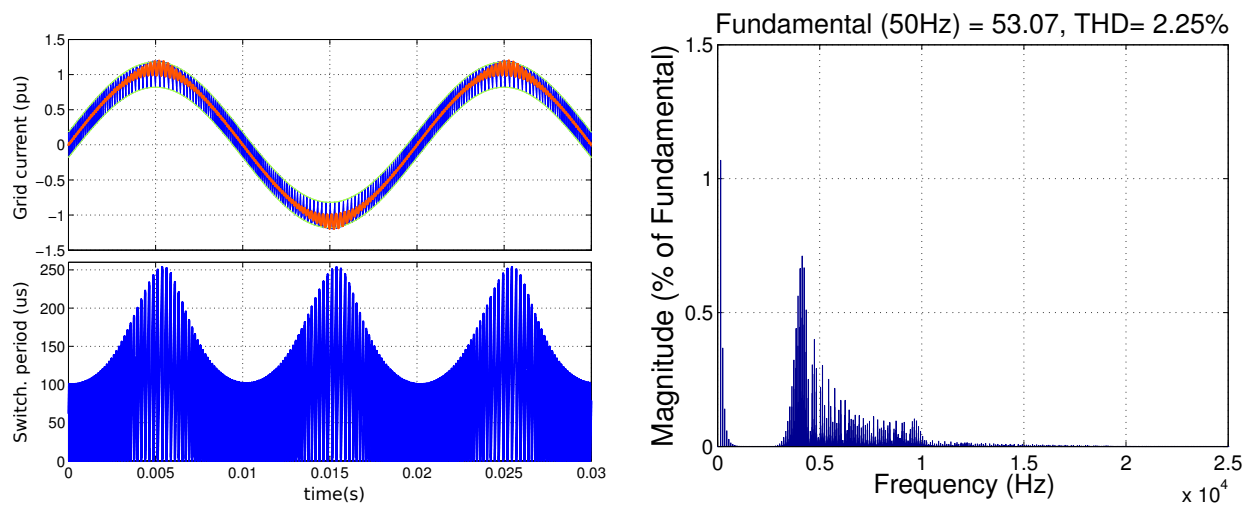

(b) $c=9 . V_{g}=220 V$.
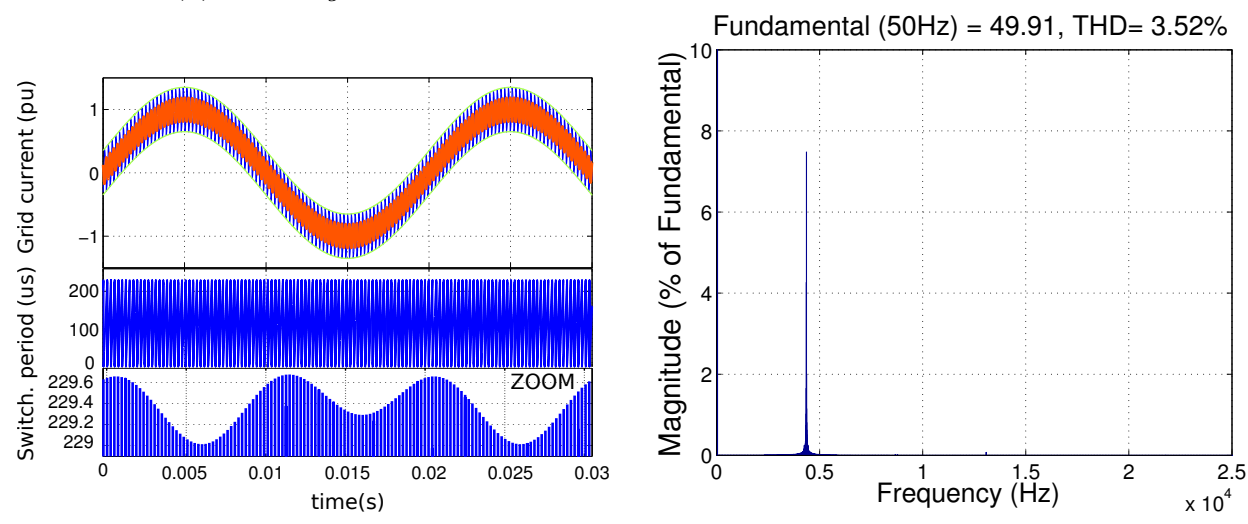

(c) $c=17.5 . V_{g}=0 V$.

Figure 17: Proposed system switching behaviour for different hysteresis bands. Left column figures: Top: Current controller response. $i_{1}$ (red line), $y=G_{p}(u)$ (blue line), and hysteresis bands (light green lines). Bottom: Switching period of the relay element. Right column figures: Current spectrum modulus. 
function from the transfer function relating the LCL grid-side current and converter output voltage. To that end a parallel compensator is designed.

The paper proposes a design process based on the duality existing between negative feedback and positive feedforward. The locus of the perturbed relay system (LPRS) has proved to be a valuable tool for analysis and design purposes. Particularly important is the information it gives about system orbital stability. The system shows stable behaviour for any orbital frequency above a minimum stability value. This minimum operating frequency is given by the LCL resonance frequency in a similar way it does in traditional linear compensation systems.

To illustrate the compensator design process, the paper has presented an 415 example design that has been tested under simulation setup. The paper has shown the correct behaviour of the system under grid voltage dips, reference step changes, grid harmonics, DC bus voltage oscillation and grid inductance $100 \%$ uncertainty conditions.

In cases where the natural behaviour is not good enough, the paper has proposed a grid feedforward compensation method that allows to practically reject all grid disturbances. In the same direction the paper describes other compensator possibilities that arise when considering the low bandwidth LPRS equivalent of the relay element.

Relay feedback controllers have represented for decades an alternative to 425 linear schemes in grid VSC converters for their advantages in certain scenarios: no need of analog-to-digital acquisition devices nor digital signal processor, nor modulators; natural good dynamics and robustness. Good proof of it is its still common use in that applications that, when controlled by these methods, are, by default, stable: L filter connection and LCL connection, with converter current 430 $i_{2}$ feedback, machine control, etc. In this sense, this article opens the possibility to also use this schema in LCL connection with grid current feedback, which is unstable by default. The obtained results seem to be promising, encouraging the authors to perform further investigations on interesting topics as, for example, a more systematic design procedure including, among other criteria, robustness 
specifications.

\section{Acknowledgment}

The work of Robert Griñó was partially supported by the Government of Spain through the Ministerio de Economía y Competitividad Project DPI201341224-P and by the Generalitat de Catalunya through the Project 2014 SGR 267. The work of Santiago Cóbreces was partially supported by the Government of Spain through the Ministerio de Economia y Competitividad Project ENE2014-57760-C2-2-R and by the Comunidad de Madrid through the Project S2013-ICE-2933.

\section{References}

[1] B.D.O. Anderson, S. Vongpanitlerd, Network Analysis and Synthesis: A Modern Systems Theory Approach, Dover Publications, 2006.

[2] K.J. Astrom, Oscillations in systems with relay feedback, in: Adaptive Control, Filtering, and Signal Processing, Springer, 1995, pp. 1-25.

[3] V. Blasko, V. Kaura, A novel control to actively damp resonance in input LC filter of a three-phase voltage source converter, IEEE Trans. Ind. Applicat. 33 (1997) 542-550.

[4] I. Boiko, Discontinuous Control Systems. Frequency-Domain Analysis and Design, Birkhäuser, 2009.

[5] B.K. Bose, An adaptive hysteresis-band current control technique of a voltage-fed PWM inverter for machine drive system, IEEE Trans. Ind. Electron. 37 (1990) 402-408.

[6] S. Cobreces, E. Bueno, F.J. Sanchez, F. Huerta, P. Rodriguez, Influence analisys of the effects of an inductive-resistive weak grid over L and LCL filter current hysteresis controllers, in: Proceedings of the 2007 European Power Electronics Conference, IEEE, Aalborg, Denmark, 2007, pp. 1-10. 
[7] M. Depenbrock, Direct self-control (DSC) of inverter-fed induction machine, IEEE Trans. Power Electron. 3 (1988) 420-429.

[8] R. Gessing, Parallel compensator for continuous and relay control systems with difficult plants, in: Proceedings of the 2007 American Control Conference. ACC'07, IEEE, New York. USA., 2007, pp. 5810-5815.

[9] S.K. Gudey, R. Gupta, Reduced state feedback sliding-mode current control for voltage source inverter-based higher-order circuit, IET Power Electron. 8 (2015) 1367-1376.

[10] X. Hao, X. Yang, T. Liu, L. Huang, W. Chen, A sliding-mode controller with multiresonant sliding surface for single-phase grid-connected vsi with an lcl filter, IEEE Trans. Power Electron. 28 (2013) 2259-2268.

[11] D.G. Holmes, R. Davoodnezhad, B.P. McGrath, An improved three-phase variable-band hysteresis current regulator, IEEE Trans. Power Electr. 28 (2013) 441-450.

475 [12] Z. Iwai, I. Mizumoto, Realization of simple adaptive control by using parallel feedforward compensator, Int. J. Contr. 59 (1994) 1543-1565.

[13] H. Kaufman, I. Bar-Kana, K. Sobel, Direct Adaptive Control Algorithms: Theory and Applications, Springer Verlag, 1998.

[14] B.H. Kwon, T.W. Kim, J.H. Youm, A novel SVM-based hysteresis current controller, IEEE Trans. Power Electron. 13 (1998) 297-307.

[15] L. Malesani, P. Tenti, A novel hysteresis control method for currentcontrolled voltage-source PWM inverters with constant modulation technique., IEEE Trans. Ind. Applicat. 26 (1990) 88-92.

[16] J.R. Massing, H. Pinheiro, Adaptive current control of grid-connected VSC with LCL-filters using parallel feedforward compensation, in: Proceedings of the 36th Annual Conference on IEEE Industrial Electronics SocietyIECON 2010, IEEE, pp. 3185-3191. 
[17] G. Pfaff, A. Weschta, A.F. Wick, Design and experimental results of a brushless AC servo drive, IEEE Trans. Ind. Applicat. IA-20 (1984) 814-

[18] I. Rusnak, I. Barkana, SPR and ASPR untangled, in: Proceedings of the 6th IFAC Symposium on Robust Control Design, ROCOND'2009, IFAC, Haifa, Israel, 2009, pp. 16-18.

[19] I. Rusnak, I. Barkana, The duality of parallel feedforward and negative feedback, in: Proceedings of the 2012 IEEE 27th Convention of Electrical \& Electronics Engineers in Israel (IEEEI), pp. 1-4.

[20] L.A. Serpa, J.W. Kolar, S. Ponnaluri, P.M. Barbosa, A modified direct power control strategy allowing the connection of three-phase inverter to the grid through LCL filters, IEEE Trans. Ind. Applicat. 43 (2007) 1388 1400.

[21] I. Takahashi, T. Noguchi, A new quick-response and high-efficiency control strategy of an induction motor, IEEE Trans. Ind. Applicat. IA-22 (1986) 820-827.

[22] Y.Z. Tsypkin, Relay Control Systems, Cambridge University Press, 1984.

[23] Z. Xianping, L. Yaxi, P. Lei, et al., Analysis and design of lcl type filter for three-phase voltage source rectifier, Electrotechnical Application 26 (2007) $65-67$.

[24] Q. Yao, D. Holmes, A simple, novel method for variable-hysteresis-band current control of a three phase inverter with constant switching frequency, in: Industry Applications Society Annual Meeting, 1993., Conference Record of the 1993 IEEE, IEEE, pp. 1122-1129. 